\section{Bacterial Contamination on Reusable Electrocardiograph Wires on Successive Operative Days}

\author{
Joseph G Werner ${ }^{1 *}$, Stephanie Mallery², Karina \\ Castellon-Larios ${ }^{1}$, Michael R Smith ${ }^{1}$, Kenneth Moran ${ }^{1,3}$, \\ Anthony R Lopez ${ }^{1}$ and Sergio D Bergese ${ }^{1,4}$
}

'Department of Anesthesiology, Wexner Medical Center at The Ohio State University, Columbus, Ohio, USA

${ }^{2}$ The Ohio State University Medical School, Columbus, Ohio, USA ${ }^{3}$ Residency Program Director, Department of Anesthesiology, Wexner Medical Center at The Ohio State University, Columbus, Ohio, USA

${ }^{4}$ Division Chief Neuroanesthesia, Director of Neuroanesthesia Fellowship, Director of Clinical and Neurological Research, Departments of Anesthesiology and Neurological Surgery, Columbus, Ohio, USA

\begin{abstract}
\section{Objective}

Nosocomial infections increase cost and morbidity in hospitals. Cross-contamination between subsequent patients in the operating room, with Electrocardiogram (EKG) leads being a possible mechanism of transfer. We tested the severity and frequency of EKG wire contamination to determine whether a transition to more costly disposable EKG leads was justifiable.

Design

Swabs were collected from cleaned multiuse EKG lead wires in 34 operating rooms at our Institution. They were cultured on agar plates to assess for the presence of Enterobacteriaceae, Acinetobacter, Enterococcus, Staphylococcus aureus and Staphylococcus epidermis. After obtaining Institutional Review Board approval, we retrospectively looked for patients with known positive cultures who had undergone surgery in each room on the day prior to culturing.

\section{Results}

The EKG cultures showed low levels of $S$. epidermidis on four of the samples (11\%). In addition, Enterococcus and Enterobacteriaceae were present on $11 \%$ and $5.6 \%$ of cultures respectively.

*Corresponding author: Joseph G Werner, Department of Anesthesiology, Wexner Medical Center at The Ohio State University, 410 West $10^{\text {th }}$ Avenue, Columbus, OH 43210, USA, Tel: 6142938487; Fax: 6142938153; E-mail: joseph.werner@osumc.edu

Citation: Werner JG, Mallery S, Castellon-Larios K, Smith MR, Moran K, et al., (2014) Bacterial Contamination on Reusable Electrocardiograph Wires on Successive Operative Days. J Anesth Clin Care 1: 001.

Received: June 01, 2014; Accepted: September 07, 2014; Published: September 22, 2014
Acinetobacter and $S$. aureus were not detected. Retrospective chart review of individuals who received surgery the day before culturing showed three patients with Methicillin-Resistant Staphylococcus Aureus (MRSA), one of which had bacteremia. Two patients had Enterococcus in the urine, two had Enterobacter, one had S. epidermidis, and one had Acinetobacter in the urine. Of the known exposures, only one set of lead cultures came back with low levels of contamination with enterococcus (20 CFU).

\section{Conclusions}

The low levels of microbial burden of EKG wires do not likely represent a source of cross-contamination between cases on successive OR days. In the perioperative setting at our medical center, it would cost approximately $\$ 300,000$ annually to switch to disposable leads, while the yearly cost for reusable leads is approximately $\$ 50,000$. With our cleaning techniques, reusable wires are safe and more cost effective than disposable leads.

Keywords: Bacterial contamination; EKG lead wire; OR - Operating Room

\section{Introduction}

Nosocomial infections cause unnecessary morbidity, mortality, cost, and increased length of hospital stay [1]. A longitudinal study of United States hospitals found that $32 \%$ of hospital acquired infections were preventable [2]. With an estimated additional cost of $\$ 13,973$ per infection, efforts to reduce these events are essential [3].

Transmission of bacteria can occur directly from one person to another, or indirectly through transfer via contaminated objects. The Electrocardiogram (EKG) is a standard monitoring device with leads that can be used multiple times between patients. Given that this device is non-invasive and not in-dwelling, it has been categorized as non-critical from an infectious transmission viewpoint [4]. However, there are multiple risk factors present that may later become a cause of nosocomial infections such as: patient skin is in contact with lead wires, caregivers manipulate EKG clips as well as other items necessary for patient care (including intravenous tubing and IV ports) during surgery, EKG wires are often placed near the incision site, and lastly, many cases are performed on patients with documented infections from virulent pathogens, which may lead to cross-contamination when used on subsequent cases.

One of the problems encountered with these multiuse EKG wires is that they cannot be cleaned with caustic agents due to the potential for structural degradation. Routine use of antimicrobials to clean the lead wires may contribute to the development of resistant bacterial strains [5].

We set out to further stratify Operating Room's (OR's) that may have been exposed to higher levels of contamination, which could lead to a higher risk of cross-contamination among patients. Traditionally, wounds are classified into one of four categories: clean, clean-contaminated, contaminated, and dirty. Examples of clean wounds include foreign body implantation, vascular surgery, and coronary bypass. Wounds are classified as clean-contaminated if they occur during gastro duodenal, biliary, colorectal, OB-GYN, genitourinary, or thoracic surgery. Contaminated wounds are those 
due to penetrating abdominal trauma or those in which accidental spillage during abdominal surgery occurs. Dirty wounds include abscess drainage and those with fecal contamination [6].

Anesthesia personnel have voiced concern about some noticeable residue remaining on reusable EKG clips after normal processing cleaning procedures have been applied, particularly in the area between clips. Although these clips are placed onto disposable EKG pads, personnel expressed concern over cross-contamination after touching these clips and then touching the other necessary patient care items, including IV tubing and injection ports. Our OR staff technicians has expressed difficulty in thoroughly cleaning the EKG wire leads, particularly at the distal portion in between the clips (Figure 1). This becomes challenging when trying to clean equipment while maintaining an efficient turnover between cases.

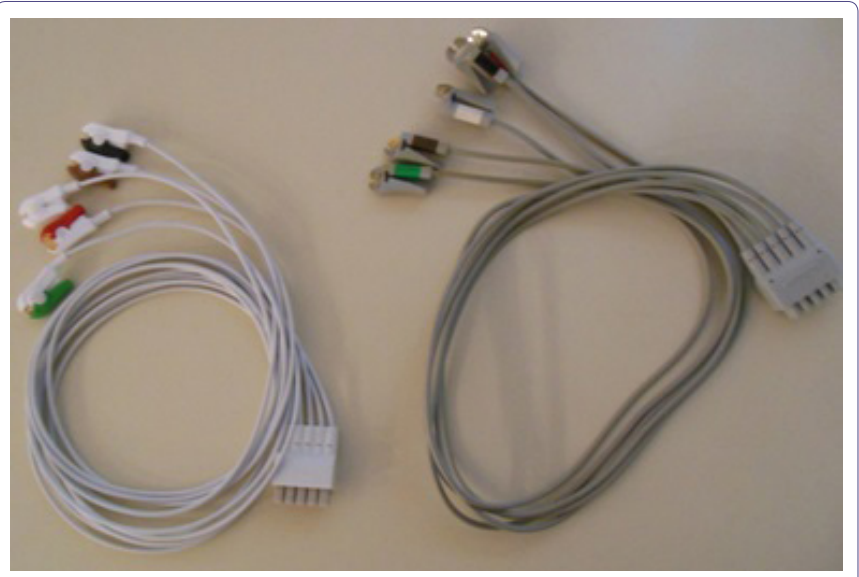

Figure 1: A new set of disposable leads (left) and a set of reusable leads Figure 1: A new set of disposable leads (left) and a set of reusable leads
immediately after cleaning (right), with the clips in the right EKG leads being identified by our operating room staff as the most difficult to clean.

Disposable EKG leads are available which could potentially reduce the incidence of cross-contamination, but would result in a $600 \%$ increase in EKG lead cost annually for our perioperative setting. Our goal was to assess the frequency and type of bacteria that may be contaminating EKG wires at our institution despite cleaning. Specifically, we monitored for residual contamination the morning after current disinfection methods were employed following the last case of the previous day.

\section{Materials and Methods}

A prospective analysis was performed to assess the rate of contamination of cleaned multiuse EKG lead wires at our institution's OR's: University Hospital, Labor and Delivery, James Cancer Center, and Ross Heart Hospital. In order to detect cross contamination from cases from the previous day, these specimens were collected in the morning before surgeries began in 34OR's throughout the medical center in the aforementioned areas. One culture was also taken from a new set of EKG lead wires directly out of the package and another after cleaning the new EKG lead wires, as control; this gave us a total of 36 cultures. Per our institutional guidelines, all patient monitoring cables used in OR's are cleaned with Sani-Cloth Plus, which are cleaning cloths that contain quaternary ammonium compounds, isopropyl alcohol and 2-butoxyethanol. The OR technicians that clean these devices put on a new pair of nitrile gloves at the beginning of the OR turnover process and wash hands with alcohol hand sanitizers upon entering and exiting the OR $[7,8]$.
Samples were obtained from the distal end of the lead wires, including the EKG pad clips. Swabs were used to collect samples from reused, cleaned EKG lead wires and were placed in Dey-Engley neutralizing buffer to neutralize any quaternary ammonium remaining on the leads. This step was performed in order to prevent residual cleaner from inhibiting growth on the cultured medium. All swabs were delivered to Richter International microbiology laboratory to enumerate bacteria in agar plates. These swabs were vortexed to remove viable bacteria off of the surface of the swabs. The following media were used to grow bacteria:

- Mannitol Salt agar $\left(37^{\circ} \mathrm{C}\right.$ for $\left.42-48 \mathrm{~h}\right)$ - Staphylococcus aureus and S. epidermidis

- Violet Red Bile Glucose agar $\left(37^{\circ} \mathrm{C}\right.$ for $\left.42-48 \mathrm{~h}\right)$ - Enterobacteriaceae

- Minimal Salt Acetate agar $\left(30^{\circ} \mathrm{C}\right.$ for $\left.48 \mathrm{~h}\right)$ [9] - Acinetobacter

- Enterococcus agar $\left(37^{\circ} \mathrm{C}\right.$ for $\left.42-48 \mathrm{~h}\right)$ - Enterococcus

This laboratory reported that the limit of detection was 10 Colony-Forming Units (CFU) per swab.

After obtaining Institutional Review Board (Office of Responsible Research Practices Biomedical Sciences Institutional Review Board) approval, we retrospectively analyzed records of all patients who underwent surgery in the OR the previous day in order to identify those with known positive cultures for pathogens requiring contact isolation precautions. All patients' records and personal information were anonymized and de-identified prior to data analysis.

\section{Results}

There were a total of 34 rooms in which swabs and cultures were performed. Positive cultures and the OR's wound classification were identified and listed in (Table 1). There were a total of ten positive cultures. Since not all cases in a given room fell under the same wound classification, only the dirtiest wound classification was considered.

\begin{tabular}{|l|c|l|}
\hline Wound Class & $\begin{array}{l}\text { Total \# of Operating } \\
\text { Rooms }\end{array}$ & $\begin{array}{l}\text { Microorganisms detected and } \\
\text { number of positive cultures }\end{array}$ \\
\hline Clean & 5 & $\begin{array}{l}\text { S. epidermidis (1) } \\
\text { Enterococcus (1) }\end{array}$ \\
\hline Clean-contaminated & 22 & $\begin{array}{l}\text { S. epidermidis (1) } \\
\text { Enterobacteriaceae (1) } \\
\text { Enterococcus (2) }\end{array}$ \\
\hline Contaminated & 1 & None \\
\hline Dirty & 6 & $\begin{array}{l}\text { S. epidermidis (2) } \\
\text { Enterobacteriaceae (1) } \\
\text { Enterococcus (1) }\end{array}$ \\
\hline
\end{tabular}

Table 1: Incidence of wound classification between operating rooms, and it's correlation with positive cultures on EKG wire leads.

Results of the cultures and pathogen exposure of patients who underwent surgery on the previous day are shown on (Table 2). Our chart review revealed a total of nine patients with positive bacterial cultures that also had contact with the rooms selected for the study on the day prior to swab collection. Three patients had MRSA, one of which had bacteremia. Two patients had Enterococcus in the urine, two had Enterobacter, one had S. epidermidis, and one had Acinetobacter in the urine. Of the known exposures, only one set of leads grew the same pathogen. This EKG lead culture showed low levels of contamination with Enterococcus (20 CFU).

The limit of detection was 10 Colony-Forming Units (CFU) per swab. The cultures showed low levels of $S$. epidermidis on four of the samples (11\%). Low levels of contamination with Enterococcus and Enterobacteriaceae were present on four samples (11\%) and two samples (5.6\%) of EKG leads respectively. Acinetobacter and S. aureus were not detected on any swabs. 
Citation: Werner JG, Mallery S, Castellon-Larios K, Smith MR, Moran K, et al., (2014) Bacterial Contamination on Reusable Electrocardiograph Wires on Successive Operative Days. J Anesth Clin Care 1: 001.

- Page 3 of $4 \cdot$

\begin{tabular}{|c|c|c|c|c|c|l|}
\hline OR & S.aureus (CFU) & S.epidermidis(CFU) & Enterobaceriaceae (CFU) & Acinetobacter (CFU) & Enterococcus (CFU) & Exposure \\
\hline 2 & $<10$ & 10 & $<10$ & $<10$ & $<10$ & None \\
\hline 12 & $<10$ & $<10$ & 40 & $<10$ & $<10$ & None \\
\hline 14 & $<10$ & $<10$ & 40 & $<10$ & $<10$ & None \\
\hline 15 & $<10$ & $<10$ & $<10$ & $<10$ & $\begin{array}{l}\text { Methicillin-Resistant } \\
\text { S.Aaureus(MRSA) }\end{array}$ \\
\hline 17 & $<10$ & $<10$ & $<10$ & $\begin{array}{l}\text { EnterobacterAcinetobacter } \\
\text { Enterococcus } \\
\text { S. epidermidis }\end{array}$ \\
\hline 19 & $<10$ & $<10$ & $<10$ & $<10$ & None \\
\hline 20 & $<10$ & 10 & $<10$ & $<10$ & $<10$ & $\begin{array}{l}\text { MRSA } \\
\text { Enterobacter }\end{array}$ \\
\hline 21 & $<10$ & 100 & $<10$ & $<10$ & $<10$ & None \\
\hline 23 & $<10$ & $<10$ & $<10$ & $<10$ & $<10$ & MRSA \\
\hline 31 & $<10$ & 220 & $<10$ & $<10$ & 10 & None \\
\hline 32 & $<10$ & $<10$ & $<10$ & $<10$ & 10 & None \\
\hline
\end{tabular}

Table 2: Significant culture results and exposure to known pathogens of patients cared for in specific operating rooms on the day prior to sampling. OR's not listed had no positive cultures.

\section{Discussion}

Our results demonstrate that the microbial burden of EKG wires and clips is low on consecutive days in the OR. Possible explanations for this are the implementation of a hand hygiene policy, as well as the methods and agents used for disinfection. According to our Institution's policy, alcohol based hand rub is recommended for routine decontamination of hands for all clinical indications $[7,8]$. This is applied upon entering a patient's bay/OR, prior to donning clean gloves, after glove removal, and upon exiting the patient's bay/OR. Internal results of several audits per year show increasing compliance with this policy since 2001 with greater than $90 \%$ compliance across the entire Institution as of 2013 [10].

In contrast to our findings, a multicenter study of EKG wire contamination was performed by Albert et al., [11] in four hospitals across the United States. Cleaned wires in critical care units, telemetry units, emergency departments, and operating rooms were cultured. Of 226 cultures, $62.8 \%$ contained at-risk or potential risk bacteria, which included S.epidermidis (29.9\%), S.haemolyticus (4\%), S.aureus (1\%), and Enterococcus faecium (1.5\%). Community hospitals had higher incidences than urban sites. This study, unfortunately doesn't describe the cleaning process employed in their standard cleaning of EKG leads nor does it describe employing a quaternary ammonium buffer during sample collection. Therefore it seems likely that other agents were used for cleaning EKG leads for their study. In 1996, a case of vancomycin-resistant enterococci led to a 13-month outbreak in a Texas hospital burn unit. Rigorous culturing and pulsed field gel electrophoresis identified several environmental sources of vancomycin-resistant enterococci. Once proper decontamination was performed, cultures in the following five weeks were negative until the outbreak recurred. Through further cultures and molecular sequencing, the bacteria were traced back to a lead wire [12].

Dr. Brookmeyer of University of Wisconsin Hospital cultured 100 random lead wires after cleaning, immediately before use on a new patient. He found that $77 \%$ were contaminated with antibiotic resistant pathogens, with $67 \%$ contaminated with MRSA [13]. Once again, no comment on cleaning techniques and agents employed. The use of quaternary ammonium compounds as a disinfectant can explain the large discrepancy in culture results between our data and previous studies.
Our results contradict previous studies of EKG lead contamination, and demonstrate that appropriate disinfection can eliminate pathogens. As (Table 2) shows, OR17 had two patients with Enterococcus infections (one with $>100,000 \mathrm{CFU} / \mathrm{mL}$ ) on the previous day, and the swabbed culture revealed only 20 CFU. Furthermore, of all the positive cultures obtained from our swabs, this was the only room that had a known exposure with the same pathogen on the previous day. From a total of 9 positive OR's (28\%), $44 \%$ of the positive lead cultures came from rooms that had cases with a dirty wound classification. Although the CFU were low in these rooms, this could represent a significant finding, and future studies should be performed in order to see if there is a meaningful correlation between this low level of bacterial contamination and postoperative infections. It is also important to note that even though the levels of contamination were low and likely don't represent a major source of cross-contamination, they still may present a clinical risk. This is especially true for immunocompromised patients. Surveillance for postoperative infections is part of our standard process for quality improvement. It is often difficult to isolate the single source of transmission as patients come into contact with many potential vectors for microorganisms during their hospital stay. In order to examine the influence of EKG lead contamination on postoperative infection, hundreds if not thousands of cultures would need to be performed to isolate potential EKG related sources of bacterial transmission and correlate them to specific patient infections. This was beyond the scope of this study as our goal was to demonstrate whether EKG leads carried a large bacterial load from one OR day to the next in order to make an informed decision about implementation of disposable EKG leads.

This study was designed to detect pathogens that are most likely to cause wound infections and also to quantify the amount of contamination present. The lower limits of our detection were $10 \mathrm{CFU}$. We could have used culture media enrichment to make our detection more sensitive (as low as $1 \mathrm{CFU}$ ), however we would not have been able to ascertain the amount of contamination present if this method was employed.

The use of disposable medical devices has lowered infection rates in many settings and may decrease hospital costs depending on item cost and utilization. Manufacturers of disposable wires cite high bacterial burden, but our evidence failed to demonstrate that EKG leads, when cleaned appropriately, are a major source of 
cross-contamination in the perioperative setting. One possible explanation for these contrasting results to previously published studies is that our study was conducted in the operating rooms whereas the majority of other studies were conducted on various hospital wards, including the intensive care unit, where leads are not likely to be cleaned as frequently. EKG leads in the OR may be cleaned three to five times per day, as compared to on the hospital wards, which only are cleaned two to three times per week. Another possible explanation for our results is that our EKG cultures were taken in the morning after the leads were cleaned and exposed to air for up to twelve hours.

At our institution, it would cost approximately $\$ 300,000$ annually to switch to disposable leads, while the yearly cost for reusable leads is just under $\$ 50,000$. Further studies should be done to assess the risk of cross-contamination with immediate use of cleaned reusable leads as having the EKG leads exposed to air overnight might itself be bactericidal.

Future studies should also be performed to look at other disinfection techniques that might diminish bacterial contamination on reusable EKG leads. One possible technique being UV light disinfection. Future studies on this technique would need to determine optimum duration of UV light exposure as well as any possible degradation to equipment. This might be particularly applicable as quaternary ammonium cleaning compounds are not effective against spore-forming Clostridium bacteria whereas UV light is effective. Future studies can also look at the prevalence of Bacillus and Clostridium bacteria as a source of cross contamination. Lastly, typing of microorganisms could be explored in future studies to better enable determination of the source of patient infection.

Our results show that reusable EKG wires are safe and that the level of cross-contamination on consecutive days of surgeries is low. Although definitive data regarding low-level cross contamination and its contribution to postoperative infections is lacking, the bacterial load on the cleaned multiuse EKG leads is low at the beginning of the workday and it seems unlikely to be a significant source of postoperative infection. Therefore, it seems reasonable to conclude that the $600 \%$ increase in annual cost for the use of disposable single use EKG leads is not justified.

\section{Acknowledgements}

Funded by Dominic and Lisa Mandalfino Memorial Fund in Anesthesiology.
We would like to thank Julie E Mangino, MD and Suren Soghomonyan, MD for their critical reviews of this manuscript. Additionally we would like to thank Dr. Edward Richter Ph.D and Dr. Jessica Dyszel, Ph.D for their guidance and input regarding the sampling and microbiology techniques.

\section{References}

1. Harbarth S, Sax H, Gastmeier P (2003) The preventable proportion of nosocomial infections: an overview of published reports. J Hosp Infect 54: 258266.

2. Haley RW, Culver DH, White JW, Morgan WM, Emori TG, et al. (1985) The efficacy of infection surveillance and control programs in preventing nosocomial infections in US hospitals. Am J Epidemiol 121: 182-205.

3. Stone PW, Larson E, Kawar LN (2002) A systematic audit of economic evidence linking nosocomial infections and infection control interventions: 19902000. Am J Infect Control 30: 145-152.

4. Lestari T, Ryll S, Kramer A (2013) Microbial contamination of manually reprocessed, ready to use ECG lead wire in intensive care units. GMS Hyg Infect Control 8: 07.

5. Carson RT, Larson E, Levy SB, Marshall BM, Aiello AE (2008) Use of antibacterial consumer products containing quaternary ammonium compounds and drug resistance in the community. J Antimicrob Chemother 62: 1160-1162.

6. Lowry SF (2005) Learning Surgery: The Surgery Clerkship Manual. Springer, New York.

7. Hand Hygiene Policy 01-03. Department of Clinical epidemiology. The Ohio State University Wexner Medical Center.

8. Aseptic Technique Policy 08-13. University Hospitals Perioperative. Policies and Procedures. The Ohio State University Wexner Medical Center.

9. Berlau J, Aucken H, Malnick H, Pitt T (1999) Distribution of Acinetobacter species on skin of healthy humans. Eur J Clin Microbiol Infect Dis 18: 179183.

10. Hand hygiene unit physician performances on the rise.

11. Albert NM, Hancock K, Murray T, Karafa M, Runner JC, et al. (2010) Cleaned, ready-to-use, reusable electrocardiographic lead wires as a source of pathogenic microorganisms. Am J Crit Care 19: 73-80.

12. Falk PS, Winnike J, Woodmansee C, Desai M, Mayhall CG (2000) Outbreak of vancomycin-resistant enterococci in a burn unit. Infect Control Hosp Epidemiol 21: 575-582.

13. Jancin B (2004) Antibiotic-Resistant Pathogens Found on $77 \%$ of ECG Lead Wires. Cardiol News 2: 14. 